\title{
RESEARCH ACTIVITIES OF EPIDEMIOLOGY IN JAPAN Infectious Disease Surveillance System of Infectious Diseases in Japan
}

\author{
Hitoshi Ohshiro, Kyoko Kawamoto, and Takayuki Nose
}

\begin{abstract}
The surveillance system of infectious disease in Japan started in 1981 and has been providing useful epidemiological information on 27 communicable diseases. The system consists of medical institutions (fixed monitoring stations), institutions of hygienic sciences, health centers, local governments and the ministry of health and welfare. There are two types of information about infectious diseases. One is clinical reports of incidence cases from medical institutions, and the other is laboratory information about etiologic agents. Between health centers, local governments and the department of statistics and information in the ministry of health and welfare, information is transmitted through the on-line network. Collected information is analyzed and submitted by both local and central committees of analysis. From the epidemiological point of view, quality control of the data and integration of other sources of data would be the next goal of the system. J Epidemiol, $1996 ; 6:$ S81-S85.
\end{abstract}

surveillance, infectious disease, epidemiology, Japan

\section{BACKGROUND}

Infectious diseases were once one of the major health problems in Japan. According to the vital statistics of 1920, the top four ranked causes of deaths were all infectious diseases : pneumonia and bronchitis, gastro-enteritis, tuberculosis and influenza. More than half of the deaths were caused by these four diseases ${ }^{1)}$. After World War II, a group of communicable diseases such as cholera, dysentery, typhoid fever, paratyphoid fever, smallpox, epidemic typhus, scarlet fever, diphtheria, epidemic meningitis, plague, Japanese encephalitis and acute poliomyelitis, which have been subjected by the Infectious Disease Prevention Law because of their high incidence and mortality rates, were also the target of public health. Incidences and mortality rates of these infectious diseases have been remarkably decreased due to vaccination programs and improvements in medical techniques, hygiene and the standard of living of nations ${ }^{2)}$. Between the end of the nineteen-forties and the beginning of the ninety-fifties, the epidemics of main infectious diseases ceased and only sporadic or limited outbreaks could be seen. Instead of these severe infectious diseases, mild infectious diseases such as rubella, mumps, measles etc. which sometimes break out suddenly and can cause severe complications or sequelae, have been focused on. To monitor and control these diseases, the surveillance system of these communicable diseases started in 1981.

This surveillance system was organized by the Ministry of Health and Welfare and is supported by local governments, health centers, local institutes of hygienic sciences, medical care institutions and the Japanese medical association. Initially, the subjects of this system were 18 diseases which mainly affect children. In June 1987, the name of the system changed to "The Surveillance System of Infectious Diseases and Tuberculosis" and added 9 communicable diseases which can affect adults, such as sexually transmitted disease, tuberculosis and viral hepatitis. Table. 1 shows diseases in the present system.

\section{SURVEILLANCE SYSTEM}

There are two types of information in the surveillance system. One is incidence information from selected clinics and hospitals, and the other is laboratory information.

\section{Incidence Information}

More than 3,000 clinics and hospitals were selected to monitor infectious diseases except tuberculosis in fixed stations

Received and accepted November 21, 1995.

Department of Public Health Faculty of Medicine, Tottori University, Yonago, Japan.

Address for correspondence : Hitoshi Ohshiro, Department of Public Health Faculty of Medicine, Tottori University, 86 Nishi-machi Yonago City, 683 Japan. 
throughout Japan. The number of the fixed stations in a certain area is decided basically according to the population of the area covered by one health center in order to make incidence analysis accurate ${ }^{3)}$. Table 2 shows the number of monitoring points

Table 1. Subject disease of the surveillance system and reported number of each disease in 1993 (per fixed station)

\begin{tabular}{lr}
\hline Measles & 34,560 \\
Rubella & 147,816 \\
Chickenpox & 186,626 \\
Mumps & 92,126 \\
Pertussis & 3,669 \\
Streptococcal infection & 70,273 \\
Atypical pneumonia & 26,524 \\
Infectious diarrhea & 409,951 \\
Rotavirus infection & 87,964 \\
Hand foot and mouth disease & 92,317 \\
Erythema infectiosum & 16,997 \\
Exanthema subitum & 86,540 \\
Herpangina & 72,427 \\
Pharyngoconjunctival fever & 4,261 \\
Epidemic Keratoconjunctivitis & 13,195 \\
Acute hemorrhagic conjunctivi & 1,785 \\
Infectious meningitis & 2,362 \\
Encephalomyelitis & 241 \\
Tuberculosis & 47,437 \\
Influenza like condition & 635,913 \\
Viral hepatitis & 4,093 \\
MCLS (Kawasaki disease) & 2,698 \\
Gonorrhea & 6,715 \\
Chlamidial disease & 13,735 \\
Condyloma acuminatum & 2,826 \\
Tricomoniasis & 3,874 \\
\hline
\end{tabular}

according to size of health centers. Table 3 shows the number of fixed station. For tuberculosis, all medical institutions must report incidence cases. Incidence cases at medical institutions are reported weekly or monthly to the health division of the local government through district health centers. Three reporting forms are designed for the outpatient clinics of pediatrics and internal medicine, ophthalmology, and clinics of sexually transmitted disease respectively. This type of information is transferred to the Ministry of Health and Welfare through the on-line network managed by the ministry from 1987.

\section{Laboratory Information}

Laboratory information concerns the identified etiologic agents isolated from patient's biological samples by the local institutions of hygienic sciences and by the laboratories of hospitals and medical schools. These samples are taken at the monitoring institutions and referred to the laboratories. Results of the identification of agents are directly reported to the National Institute of Hygiene and summarized by the institute both monthly and annually. On-line system for the laboratory information has not established yet.

\section{DATA BASE AND DATA ANALISIS}

Reported information is stored as a data-base in the main frame computers at the Department of Statistics and Information in the Ministry of Health and Welfare. The database is managed by the Center for Tuberculosis and Infectious Diseases Information. This organization manages the database and summarizes the information. The laboratory and clinical information is analyzed and evaluated by the committee of analysis under the control of the Center for Tuberculosis and Infectious Diseases Information. The committee consists

Table 2. Number of monitoring points by size of health center.

\begin{tabular}{lcc}
\hline $\begin{array}{l}\text { Type of health center } \\
\text { (regional population of health center) }\end{array}$ & $\begin{array}{c}\text { Number of monitoring points } \\
\text { Pediatrics and } \\
\text { Internal Medicine }\end{array}$ & STD \\
\hline Type 1 $(250,000$ or more) & 5 & 1 \\
Type 2 (175,000-250,000) & 4 & 1 \\
Type 3 (125,000-175,000) & 3 & 1 \\
Type 4 ( 75,000-125,000) & 2 & 1 \\
Type 5 ( 30,000- 75,000) & 1 & \\
Type S (less than 30,000) & 1 & \\
\hline
\end{tabular}

Table 3. Number of monitoring points by type of institution.

\begin{tabular}{lr}
\hline Pediatrics and Internal Medicine & 2425 \\
Ophthalmology & 315 \\
Hospital & 544 \\
Sexually transmitted disease & 596 \\
Total & 3880 \\
\hline
\end{tabular}




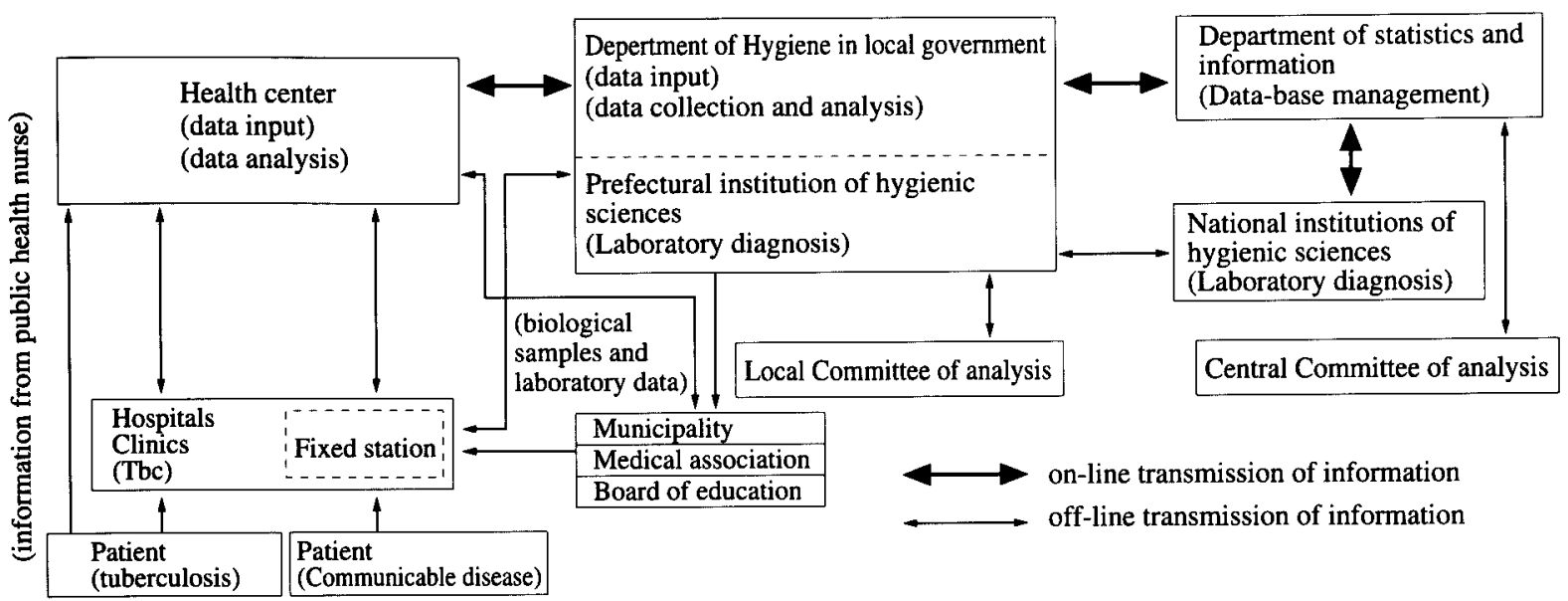

Figure 1. Schema of the surveillance system of infectious disease in Japan.

of specialists of infectious diseases and it also supervises two kinds of sub-committees, namely subgroups for information analysis and a subgroup for surveillance systems. Subgroups for information analysis consists of four groups : pediatric and internal medicine group, STD group, pathologic agents information group and tuberculosis group respectively. These subcommittees have monthly meetings to analyze the condition of outbreaks and tabulate the data which is broken down by disease, age, area and prefecture etc. and reported to local governments. These sub-committees also evaluate the surveillance system and administration for infectious disease prevention. The counterpart of each committee system exists in every prefecture in Japan. The results of analyses are reported weekly and monthly to prefectural governments and health centers through the same on-line network which transfers incidence information from health centers. The scheme of the surveillance system is shown in Fig.1.

\section{APPLICATION OF THE SURVEILLANCE DATA}

Data from surveillance systems is utilized to both predict the outbreak of communicable diseases and for epidemiological research. Using MEDLINE and the "Japana Centra Revuo Medicina", Japanese reference database, 243 reports concerned with the Surveillance system of infectious diseases in Japan were searched from 1985 to 1994.

These reports can be categorized as follows.

1) Routine reports from local or central government.

2) Descriptive reports about epidemics of infectious diseases.

3) Analysis or forecasting epidemics of the diseases using modeling approaches.

4) Evaluation of the surveillance system concerning quality control or implementation and application of surveillance data.

5) Introductory reports of the system.

Table 4 shows the number of published report specified by the category.

Table 4. Types of published reports from 1985 to 1994

\begin{tabular}{rrrrrrrrrrrrr}
\hline & 85 & 86 & 87 & 88 & 89 & 90 & 91 & 92 & 93 & 94 & $85-94$ \\
\hline 1 & 3 & 11 & 13 & 17 & 19 & 11 & 10 & 8 & 10 & 3 & 105 \\
2 & 3 & 11 & 8 & 8 & 3 & 7 & 5 & 4 & 8 & 4 & 61 \\
3 & 3 & 0 & 2 & 3 & 3 & 1 & 1 & 1 & 3 & 3 \\
4 & 1 & 6 & 2 & 6 & 3 & 3 & 4 & 1 & 3 & 1 & 30 \\
5 & 1 & 6 & 6 & 3 & 5 & 3 & 0 & 1 & 2 & 0 & 27 \\
Total & 11 & 34 & 31 & 37 & 33 & 25 & 20 & 15 & 26 & 11 & 243 \\
\hline
\end{tabular}

1) Routine reports from local or central government.

2) Descriptive reports about epidemics of infectious diseases.

3) Analysis or forecasting epidemics of the diseases using modeling approaches.

4) Evaluations of the surveillance system concerning quality control, implementation or application.

5) Review or introductory reports of the system. 


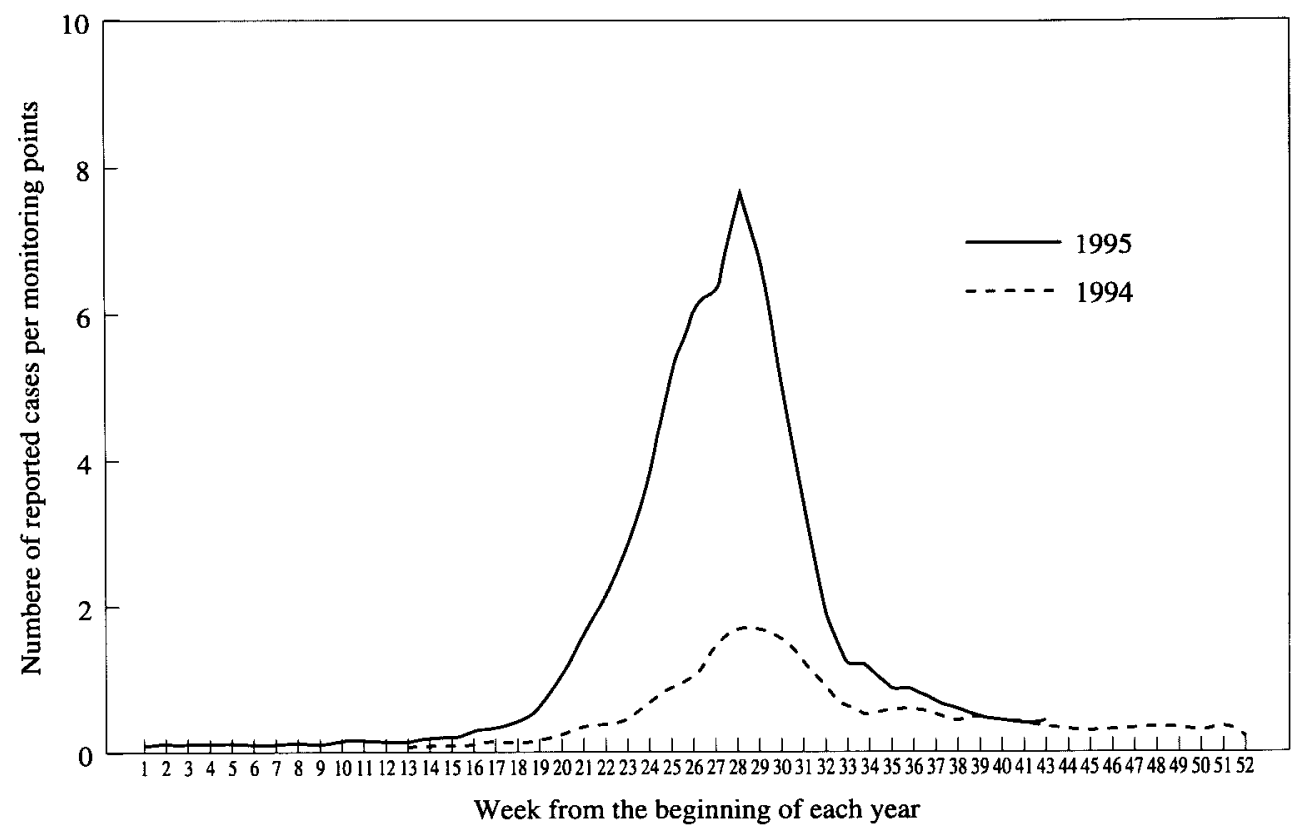

Figure 2. Incidence of hand foot and mouth disease by week from 1994-1995.

One of the latest examples of forecasting epidemics of communicable diseases is that of hand foot and mouth disease. In 1995, outbreak of hand foot and mouth disease was observed in Japan. This disease breaks out occasionally because of the change of population immunity. Since starting the surveillance system in 1981, the disease broke out in 1982, 1985, 1988 and 1990. The 1995 outbreak was predicted several months prior to its peak because of the early increace of incidence ${ }^{4}$. Fig.2 shows the pattern of incidence of the disease. The epidemics of other diseases, such as influenza, rubella, mumps, measles and streptococcal infectious disease, have been analyzed ${ }^{5,6,7,8)}$. This means that the system provides enough information to forecast the prevalence of certain diseases.

\section{PROBLEMS OF THE PRESENT SYSTEM}

Although the system provides useful information in predicting the outbreak of communicable diseases, there are some problems in the system in epidemiological analysis such as the evaluation of public health implementation for prevention, immunization programs, and regional distribution. One of the problems is the accuracy of the data. The reliability of the data depends on the number and capturing efficiency of monitoring points which cover each region. There have been several efforts to standardize the reported number in different areas in $\operatorname{Japan}^{9,10,11)}$. Another problem is that the system is not complete yet. For example, information about pathologic agents isolated from biological samples has not been transferred through the on-line network. Furthermore, it has been pointed out that the next goal of the system should be the integration of various data from other systems, such as immunity status of population or information of pathologic agents isolated in laboratories $^{2,3)}$.

\section{REFERENCES}

1. Vital statistics of Japan, 1993 (in Japanese).

2. Anonymous. The surveillance system of tuberculosis and infectious diseases, Journal of Health and Welfare Statistics 1995 ; Supplement, 42 : 157-159 (in Japanese).

3. Shiomi S. Surveillance system of Infectious diseases in Japan, Journal of Health and Welfare Statistics, 1987 ; 34 : 10-16 (in Japanese).

4. Kimura M. Infectious disease forecasting, Nikkei Medical, 1995 ; August 15. 66-67 (in Japanese).

5. Kitayama $A$, Ichimura $H$, Ohtawara $M$, Iida $K$. A Statistical Analysis on the Forecasting of Numbers of Patients in the Surveillance of Infectious Diseases, Chiba eiken houkoku, $1986 ; 10: 30-34$.

6. Nakamura Y, Yanagawa H, Nagai M. Epidemic patterns of infectious diseases from the result of the surveillance of infectious diseases in Japan, Pediatr Infect Dis J, 1988 ; $7: 262-266$.

7. Hirayama M. Surveillance of Communicable Diseases and Immunization Program, Acta Pediatric Jpn, 1988 ; 30 :127-135.

8. Murakami T, Haruki K, Seto T, Kimura T, Shibe K, Minoshiro S. Differences in influenza epidemics in 
Osaka city-epidemiologycal surveillance of infectious disease, Japanese Journal of Public Health, 1994 ; 41 : 461-465.

9. Morita M. A Study on the Examination of the Incidences of Streptococcal Infectious Diseases in the Nationwide and the Regional Surveillance Information of Infectious Diseases in Japan(2nd Report), Kansenshogaku-Zasshi, $1991 ; 65$ : 913-918.
10. Doi W. Studies on the Accuracy and Effectiveness of the Infectious Disease Surveillance System, with Regard to the Characteristics of Clinics to be Selected as Monitoring Stations, Jpn J Hyg, 1987 ; 42 : 994-1004.

11. Hamamura N. A Study of Standardization of Surveillance Data 1) Significance of "Corrected Value of Patients" and Its Application to Exanthema Subitum and Erythema Infectiosum, Kansenshogaku-Zasshi, 1993 ; 67 : 515-523. 